\title{
Baryogenesis via neutrino oscillations
}

\author{
E. Kh. Akhmedov ${ }^{(a, b)}$ V. A. Rubakov ${ }^{(c, a, d)}$ and A. Yu. Smirnov ${ }^{(a, c)}$ \\ ${ }^{(a)}$ The Abdus Salam International Centre for Theoretical Physics, I-34100 Trieste, Italy \\ (b) National Research Centre Kurchatov Institute, Moscow 123182, Russia \\ ${ }^{(c)}$ Institute for Nuclear Research of the Russian Academy of Sciences, Moscow 117312, Russia \\ ${ }^{(d)}$ Institute for Cosmic Ray Research, University of Tokyo, Tanashi, Tokyo 188, Japan
}

(March 5, 1998)

\begin{abstract}
We propose a new mechanism of leptogenesis in which the asymmetries in lepton numbers are produced through the CP-violating oscillations of "sterile" (electroweak singlet) neutrinos. The asymmetry is communicated from singlet neutrinos to ordinary leptons through their Yukawa couplings. The lepton asymmetry is then reprocessed into baryon asymmetry by electroweak sphalerons. We show that the observed value of baryon asymmetry can be generated in this way, and the masses of ordinary neutrinos induced by the seesaw mechanism are in the astrophysically and cosmologically interesting range. Except for singlet neutrinos, no physics beyond the Standard Model is required.
\end{abstract}

PACS: 98.80.Cq, 14.60.St IC/98/22, INR-98-14T hep-ph/9803255

1. The origin of the excess of baryons over anti-baryons in the Universe remains one of the fascinating problems of particle physics and cosmology. A number of mechanisms have been proposed to date to explain this asymmetry (for recent reviews see, e.g., [1]). One of the simplest possibilities, suggested by Fukugita and Yanagida [2], is that the baryon asymmetry has originated from physics in the leptonic sector. Namely, it was assumed that at temperatures well above the electroweak scale, lepton asymmetry was produced, which was then reprocessed into the baryon asymmetry by non-perturbative electroweak effects [3]-sphalerons [4]. According to ref. [2] the lepton asymmetry is generated in out-of-equilibrium, CP- and lepton number non-conserving decays of heavy Majorana neutrinos (for recent discussions see, e.g., ref. [5] and references therein).

In this Letter we propose a new realization of baryogenesis through leptogenesis which also makes use of the electroweak reprocessing of the lepton number into the baryon number. Like the Fukugita-Yanagida mechanism, our proposal requires only mild extension of the Standard Model by introducing "sterile" (i.e., electroweak singlet) heavy neutrinos. However, our mechanism of leptogenesis is entirely different from that of ref. [2]: we suggest that asymmetries in lepton numbers were generated due to oscillations of these singlet neutrinos and their interactions with ordinary matter in the early Universe. Moreover, the novel feature of our scenario is that the total lepton number is not violated in these oscillations and/or interactions; an important ingredient is separation (rather than generation) of lepton number, i.e., its redistribution between different species of singlet neutrinos.

For this reason we do not necessarily require that singlet neutrinos be Majorana particles; Dirac "sterile" neutrinos are equally suitable (and even better in some respect) for our mechanism to work. Furthermore, in our case the values of the masses and couplings of singlet neutrinos are very different from those of ref. [2].

2. Let us consider the Standard Model extended by adding three types of Majorana neutrinos $N_{a}, a=$ $A, B, C$ which interact with other particles only through their Yukawa couplings [6]. The corresponding Lagrangian can be written in the "Yukawa basis" (where the matrix of Yukawa coupling constants has been diagonalized) as follows,

$$
\mathcal{L}=\bar{N}_{R a} i \not \partial N_{R a}+h_{a} \bar{l}_{a} N_{R a} \Phi+\frac{M_{a b}}{2} N_{R a}^{T} C N_{R b}+\text { h.c. . }
$$

Here $N_{R a}$ are right-handed components of $N_{a}, h_{a}$ are (real) Yukawa coupling constants, $l_{a}$ are three independent linear combinations of usual leptonic doublets, $\Phi$ is the Higgs doublet, and $M_{a b}$ is the mass matrix.

We suggest that the baryogenesis proceeds in the following way:

(i) In the course of the evolution of the Universe, singlet neutrinos are produced through their Yukawa couplings. The production mechanism of singlet neutrinos conserves $\mathrm{CP}$, i.e. for each type equal numbers of particles and antiparticles (particles of opposite helicities in the Majorana case) are produced.

(ii) Once created, singlet neutrinos oscillate, and also interact with ordinary matter. None of these processes violates the total lepton number $L^{\text {tot }}=L+L_{A}+L_{B}+L_{C}$, where $L$ is the usual lepton number (we assume that Majorana masses are small enough, see below). However, CP is not conserved due to mixing in the singlet neutrino sector. Therefore the initially created state with individual lepton numbers $L_{A}=L_{B}=L_{C}=0$ evolves through the oscillations into a state in which $L_{A} \neq 0, L_{B} \neq 0, L_{C} \neq 0$ but still $L^{\text {tot }}=0$. That is, the total lepton number gets unevenly distributed between different species.

(iii) Singlet neutrinos communicate their lepton asymmetry to ordinary neutrinos and charged leptons through their Yukawa couplings. We assume that the Yukawa 
couplings of $N_{A}, N_{B}$ and $N_{C}$ have certain hierarchy, so that neutrinos of at least one type, $N_{A}$, come into thermal equilibrium before the time $t_{E W}$ at which sphalerons become inoperative (the corresponding temperature is $T_{E W} \sim 100 \mathrm{GeV}$ ) and those of at least one other type, $N_{C}$, do not equilibrate by $t_{E W}$. The neutrinos of the third type, $N_{B}$, may or may not come into thermal equilibrium by $t_{E W}$. To be specific, we discuss mostly the case when the Yukawa coupling of $N_{B}$ is relatively large so that $N_{B}$ equilibrate at temperatures exceeding $T_{E W}$ (the opposite case is treated in a similar way). In this case the lepton numbers $L_{A}$ and $L_{B}$ are communicated to the ordinary leptons before $t_{E W}$ whereas $L_{C}$ is not. Therefore (a fraction of) $L_{A}+L_{B}$ is reprocessed into baryon asymmetry by electroweak sphalerons; the lepton number $L_{C}$ is transfered to the active leptons only after the sphalerons have already switched off, so it has no effect on the baryogenesis.

Let us stress that if not for the sphaleron freezing effect, no baryon asymmetry would have been obtained: all three singlet neutrino species eventually transform into ordinary leptons, and since $L^{\text {tot }}=0$, no net lepton charge would have been generated in the sector of ordinary leptons. Hence, the requirement that $N_{C}$ do not get into thermal equilibrium before $t_{E W}$ is crucial for our mechanism.

3. The system of three types of singlet neutrino of a given momentum $k(t) \propto T(t)$, interacting with cosmic plasma, is described by $3 \times 3$ density matrix $\rho(t)$ which obeys the evolution equation [7]

$$
i \frac{d \rho}{d t}=[\hat{H}, \rho]-\frac{i}{2}\{\Gamma, \rho\}+\frac{i}{2}\left\{\Gamma^{p}, 1-\rho\right\}
$$

Here $\hat{H}$ is Hermitean effective Hamiltonian, $\Gamma$ and $\Gamma^{p}$ are destruction and production rates, respectively, and we have neglected the relaxation due to elastic scattering, see below. In what follows we will use the approximation of Boltzmann statistics for order-of-magnitude analysis; in this approximation the last term in eq.(11) becomes merely $i \Gamma^{p}$.

In the Yukawa basis one has at $T \gg M_{a}$

$$
\hat{H}=V(t)+U \frac{\hat{M}^{2}}{2 k(t)} U^{\dagger} .
$$

Here $U$ is the mixing matrix which relates the Yukawa states and the mass eigenstates $N_{i}(i=1,2,3)$ : $N_{a}=U_{a i} N_{i}$ (we assume that mixing is small, and choose the standard parametrization for $U$ [8]); $\hat{M}^{2}=$ diag $\left(M_{1}^{2}, M_{2}^{2}, M_{3}^{2}\right)$ is the matrix of mass eigenvalues; $k(t)$ is the neutrino momentum which depends on time due to the expansion of the Universe. The CP violation in the system is described by the CP-odd phase $\delta$ in the mixing matrix $U$.

The medium effects are described by real potential $V$ and rates $\Gamma$ and $\Gamma^{p}=\exp (-k / T) \Gamma$ [9] whose matrices are diagonal in the Yukawa basis,

$$
V=\operatorname{diag}\left(V_{A}, V_{B}, V_{C}\right), \Gamma=\operatorname{diag}\left(\Gamma_{A}, \Gamma_{B}, \Gamma_{C}\right)
$$

The potentials $V_{a}$ are due to the coherent forward scattering processes, the main contribution coming from the 1loop self energy diagrams with ordinary lepton and Higgs doublets in the intermediate states. For our estimates we use their thermal averages which at temperatures above $T_{E W}$ are 10

$$
V_{a}=\frac{1}{8} h_{a}^{2} T .
$$

Consider now the rates $\Gamma_{a}$. We will be interested in the temperatures far exceeding the masses of singlet neutrinos. Therefore the rates of the $1 \leftrightarrow 2$ reactions which correspond to the absorptive parts of the self energy diagrams are suppressed by the factor $M_{a} / T$ and $2 \leftrightarrow 2$ reactions are more important. The main contributions to $\Gamma_{a}$ come from the Higgs exchange reactions $Q_{L} N_{R a} \leftrightarrow t_{R} l_{a}$, $t_{R}^{c} N_{R a} \leftrightarrow Q_{L}^{c} l_{a}$ and $l_{a}^{c} N_{R a} \leftrightarrow t_{R} Q_{L}^{c}$ where $Q_{L}$ is the third-generation quark doublet. The result [11] for the average destruction rates at $T \gg M_{a}$ (corrected to include the color factor for quarks) is

$$
\Gamma_{a} \simeq \frac{9 h_{t}^{2}}{64 \pi^{3}} h_{a}^{2} T .
$$

Here $h_{t} \simeq 1$ is the top quark Yukawa coupling. The rates of the elastic $2 \rightarrow 2$ scattering processes are proportional to $h_{a}^{2} h_{b}^{2}$ instead of $h_{a}^{2} h_{t}^{2}$. We will need very small Yukawa couplings of singlet neutrinos, so the elastic processes can be safely neglected.

At late times the lepton asymmetry is stored in the least interacting species of singlet neutrino, which at $t \sim t_{E W}$ coincides with the mass eigenstate $N_{3} \approx N_{C}$. The conditions that $N_{1}\left(\approx N_{A}\right)$ and $N_{2}\left(\approx N_{B}\right)$ come into thermal equilibrium before the time $t_{E W}$, while $N_{3}$ do not, are

$$
\Gamma_{1,2}\left(T_{E W}\right)>H\left(T_{E W}\right), \quad \Gamma_{3}\left(T_{E W}\right)<H\left(T_{E W}\right),
$$

where $H(T)=T^{2} / M_{\mathrm{Pl}}^{*}$ is the Hubble parameter, $M_{\mathrm{Pl}}^{*} \equiv$ $M_{\mathrm{Pl}} / 1.66 \sqrt{g_{*}} \simeq 10^{18} \mathrm{GeV}$, and $\Gamma_{3} \approx \Gamma_{C}+s_{13}^{2} \Gamma_{A}+s_{23}^{2} \Gamma_{B}$. Here $s_{13} \equiv \sin \theta_{13}$ and $s_{23} \equiv \sin \theta_{23}$ determine the admixtures of $N_{A}$ and $N_{B}$ in $N_{3}$.

The conditions (5) translate into bounds on the Yukawa couplings,

$$
h_{A, B}^{2}>2 \cdot 10^{-14}, \quad h_{C}^{2}<2 \cdot 10^{-14},
$$

and on mixing angles,

$$
s_{13}^{2}<2 \cdot 10^{-14} h_{A}^{-2}, s_{23}^{2}<2 \cdot 10^{-14} h_{B}^{-2} .
$$

Eq. (6) implies a certain hierarchy between the Yukawa couplings, which, however, need not be very strong.

4. As described above, to find the baryon asymmetry we should calculate the asymmetry $\Delta_{L}\left(t_{E W}\right)$ which was communicated to usual leptons by the time $t_{E W}$ at which 
sphalerons switch off. This asymmetry emerges because singlet neutrinos $N_{3}$ do not transfer their asymmetry to active neutrinos by the time $t_{E W}$ due to smallness of $h_{C}$, $s_{13}$ and $s_{23}$. Since the total lepton number is conserved in all processes of interest, we have $\Delta_{L}\left(t_{E W}\right)=-\Delta_{3}\left(t_{E W}\right)$ (up to a factor of order one that accounts for the distribution of the asymmetry between $B, L$ and $L_{A}+L_{B}$; we will not write this factor in formulas below), where $\Delta_{3}\left(t_{E W}\right)$ is the asymmetry stored in $N_{3}$. The asymmetry $\Delta_{3}\left(t_{E W}\right)$ can be found as follows. Let $S\left(t, t_{0}\right)$ be the evolution matrix corresponding to the operator $\tilde{H}=\hat{H}-(i / 2) \Gamma$ (notice that $S\left(t, t_{0}\right)$ is not unitary since this operator is nonHermitean). The density matrix $\rho(t)$ can be expressed through $S\left(t, t_{0}\right)$ assuming $\rho\left(t_{i}\right)=0$ where $t_{i}$ is the time at which the production of singlet neutrino begins [12]. The ratio of the number density of $N_{3}, n_{3}=\rho_{33}$, to the equilibrium density of one spin degree of freedom at time $t_{E W}$ is

$$
\frac{n_{3}\left(t_{E W}\right)}{n_{e q}\left(t_{E W}\right)}=\sum_{a, b} \int_{t_{i}}^{t_{E W}} d t_{0} \Gamma_{a}\left(t_{0}\right)\left|U_{3 b}^{\dagger} S_{b a}\left(t_{E W}, t_{0}\right)\right|^{2}
$$

The asymmetry $\Delta_{3}\left(t_{E W}\right)$ is the CP-odd part of the quantity (8).

The integration over the production time $t_{0}$ can be performed in a closed form. Indeed, the matrices $S$ and $S^{\dagger}$ obey $\partial_{t_{0}} S=i S \tilde{H}\left(t_{0}\right), \partial_{t_{0}} S^{\dagger}=-i \tilde{H}^{\dagger}\left(t_{0}\right) S^{\dagger}$. From these equations and $\Gamma=i\left(\tilde{H}-\tilde{H}^{\dagger}\right)$, one finds that $\partial_{t_{0}}\left(S S^{\dagger}\right)=S \Gamma\left(t_{0}\right) S^{\dagger}$. Using this relation one can readily perform the integration over the production time $t_{0}$ in Eq. (8),

$$
\frac{n_{3}\left(t_{E W}\right)}{n_{e q}\left(t_{E W}\right)}=1-\left[S^{M}\left(t_{E W}, t_{i}\right) S^{M}\left(t_{E W}, t_{i}\right)^{\dagger}\right]_{33} .
$$

Here $S^{M} \equiv U^{\dagger} S U$ is the evolution matrix in the mass eigenstate basis. The CP-odd part of this expression determines the asymmetry transfered to usual leptons by $t_{E W}$ (and hence the generated baryon asymmetry)

$$
\begin{aligned}
\Delta_{L}\left(t_{E W}\right) & \equiv\left(n_{L}-n_{\bar{L}}\right) / n_{\gamma}=-\Delta_{3}\left(t_{E W}\right) \\
& =\frac{1}{2} \sum_{i}\left|S_{3 i}^{M}\left(t_{E W}, t_{i}\right)\right|_{C P-o d d}^{2}
\end{aligned}
$$

where the factor $1 / 2$ accounts for two helicity states of photon. The production of singlet neutrinos starts at very early times, so we set $t_{i}=0$. Since $T_{E W}$ is much smaller than all relevant energy parameters in the problem, one can formally let $t_{E W} \rightarrow \infty$ in actual calculations.

5. The lepton asymmetry is produced mainly at the epoch $t_{L}$ when the differences of the eigenvalues of the Hamiltonian $\Omega_{i j} \equiv \Omega_{i}-\Omega_{j}$ become of order of the Hubble parameter $H$ : $\Omega_{i j}\left(t_{L}\right) \sim 1 / t_{L}$. Indeed, at $t \ll t_{L}$ the elements of $\rho(t)$ essentially stay constant, whereas at $t \gg$
$t_{L}$ they undergo fast oscillations and due to averaging effects the asymmetry is strongly suppressed.

In what follows we will present the results for the most interesting range of the parameter space where the mass differences are relatively large, $\Delta M^{2} \gg\left(h_{a b}^{2} / 8\right)^{3} M_{P l}^{*}{ }^{2}$ $\left(h_{a b}^{2} \equiv h_{a}^{2}-h_{b}^{2}\right), \Delta M^{2}$ being the typical value of $\Delta M_{i j}^{2}$. In this case the mass terms dominate over potentials in $\hat{H}$ at the epoch $t_{L}$, so that $\Omega_{i j} \sim \Delta M_{i j}^{2} / 2 T$ and the leptogenesis temperature is $T_{L} \equiv T\left(t_{L}\right) \sim\left(M_{P l}^{*} \Delta M_{i j}^{2}\right)^{1 / 3}$. At $t=t_{L}$ we have

$$
\frac{\left|V_{a b}\right|}{H}=\frac{h_{a b}^{2}}{8}\left(\frac{M_{P l}^{* 2}}{\Delta M^{2}}\right)^{1 / 3} \equiv \lambda \ll 1 .
$$

This means that the potentials $V_{a}$ and rates $\Gamma_{a}$ can be treated perturbatively with $\lambda$ being the expansion parameter. The lepton asymmetry (9) appears in the third order of perturbation theory, and therefore is suppressed by the cube of $\lambda$. This can be seen in the mass eigenstate basis, where the potential has the form $U^{\dagger} V U$. Indeed, $\Delta_{L}$ is a CP - violating observable, so it should be proportional to the invariant $J=s_{12} c_{12} s_{13} c_{13}^{2} s_{23} c_{23} \sin \delta$; this invariant can be collected from $\left[U^{\dagger} V U\right]^{3}$ only. It is clear from eqs. (8) and (9) that $\Delta_{L}$ vanishes in the limit $\Gamma \rightarrow 0$, so one expects it to be proportional also to $\sin \phi \equiv \Gamma_{a} / 2 V_{a} \simeq 2 \cdot 10^{-2}$. Therefore, up to a numerical constant we have an estimate

$$
\Delta_{L} \sim J \lambda^{3} \sin \phi
$$

The calculations are simplified if $\left|\Delta M_{13}^{2}\right| \ll$ $\left|\Delta M_{12}^{2}\right|,\left|\Delta M_{23}^{2}\right|$. In this case, using Eq. (9), we obtain

$$
\Delta_{L} \simeq \frac{\left[\Gamma\left(\frac{1}{3}\right)\right]^{3}}{384} J \sin \phi \frac{h_{A C}^{2} h_{A B}^{2} h_{B C}^{2} \cdot M_{P l}^{* 2}}{\left(\Delta M_{13}^{2}\right)^{1 / 3}\left(\Delta M_{12}^{2}\right)^{2 / 3}}
$$

where $\Gamma(1 / 3) \simeq 2.68$. The estimate (11) as well as the formula (12) are valid both in the case when $N_{2}$ equilibrate before $t_{E W}$ and in the opposite case.

The asymmetry increases when the parameter $\lambda$ approaches 1 , i.e., the maximal effect for given $h_{a}$ is expected when $\Delta M^{2} \sim\left(h_{a b}^{2} / 8\right)^{3} M_{P l}^{* 2}$. Notice, however, that these and smaller values of $\Delta M^{2}$ correspond to singlet neutrinos strongly degenerate in mass.

6. Let us present constraints on the parameters of singlet neutrinos and discuss the value of the asymmetry.

(i) To be consistent with the standard mechanism of nucleosynthesis, all singlet neutrinos, including the most weakly interacting one $N_{3}$, should decay before the nucleosynthesis epoch. The decay of $N_{3}$ at $T \ll T_{E W}$ occurs due to its mixing with ordinary neutrino. Requiring that the decay rate of $N_{3}$ exceeds the inverse lifetime of the Universe at temperatures of order of a few $\mathrm{MeV}$, and recalling Eq. (6), we obtain a lower bound on the mass, $M_{3} \gtrsim 1 \mathrm{GeV}$. Alternatively, for $M_{3} \gg 1$ $\mathrm{GeV}$, we have a lower bound on the Yukawa constant, $h_{3}^{2} \gtrsim 10^{-16}\left(1 \mathrm{GeV} / M_{3}\right)^{3}$ where $h_{3}^{2} \simeq h_{C}^{2}+s_{13}^{2} h_{A}^{2}+s_{23}^{2} h_{B}^{2}$. 
(ii) If the mass of $N_{3}$ is close to $1 \mathrm{GeV}$, and/or the Yukawa couplings of $N_{A}$ and $N_{B}$ are close to the bound (6), the decays of singlet neutrinos may lead to the reheating of the Universe after the electroweak epoch (but before nucleosynthesis), and hence to the dilution of the baryon asymmetry. This reheating is rather modest, however: given the constraints already imposed, the entropy density may increase at most by a factor of 10 . In this case the baryon asymmetry produced before the reheating should be an order of magnitude larger than the observed one.

(iii) Baryon and lepton asymmetries should not be washed out before $T=T_{E W}$ by the Majorana mass itself. At $T \gg M_{A}$ the lepton number equilibration rate is suppressed with respect to the lepton charge conserving rate $\Gamma_{A}$, given by Eq. (4), by a factor $M_{A}^{2} / T^{2}$, so we have to require that $\Gamma_{A}\left(T_{E W}\right)\left(M_{A}^{2} / T_{E W}^{2}\right) \ll H\left(T_{E W}\right)$, and similarly for $N_{B}$. The parameters of Majorana singlet neutrinos should therefore satisfy

$$
1 \mathrm{GeV} \lesssim M_{a} \ll 100 \mathrm{GeV}, \quad h_{A}^{2}, h_{B}^{2} \ll 10^{-10} .
$$

The upper bounds here do not apply to Dirac singlet neutrinos.

The Lagrangian of the model leads, via the see-saw mechanism, to the generation of masses of the light (active) neutrinos: $m_{\nu_{a}} \equiv m_{a}=h_{a}^{2} v^{2} / M_{a}$, where $v$ is the Higgs vacuum expectation value and $\nu_{a}$ are mass eigenstates - combinations of $\nu_{e}, \nu_{\mu}$ and $\nu_{\tau}$. The constraints (6) and (13) imply that the mass of the heaviest active neutrino is in the range $m_{a}=\left(10^{-2}-10^{3}\right) \mathrm{eV}$. From the cosmological bound $m_{a} \lesssim 10 \mathrm{eV}$ we get a constraint which is somewhat stronger than Eq. (13), $h_{A}^{2} \lesssim 10^{-11}$. For the lightest active neutrino, the constraints (6) and (13) lead to $m_{C}=\left(10^{-6}-10^{-1}\right) \mathrm{eV}$. (Notice that the mixing parameters of active neutrinos are unconstrained by our scenario as they are not directly related to the mixing matrix of singlet neutrinos.)

The above constraints imply that the condition (10) is indeed satisfied in large part of the allowed parameter space. In particular, it holds in the two cases which we now turn to.

As follows from Eq. (12), in the case when two of the singlet neutrinos are relatively strongly interacting the desired lepton (and baryon) asymmetry $\Delta_{L} \sim$ (a few $) \cdot 10^{-9}$ is obtained for generic values of the parameters subject to the above constraints. For example, for $h_{A}^{2} \sim h_{B}^{2}=10^{-12}, \Delta M^{2} \sim M_{a}^{2}$ and $M_{a}=10 \mathrm{GeV}$ the correct asymmetry is generated provided that $J \gtrsim 10^{-3}$ which is certainly consistent with Eq. (]). The temperature of leptogenesis is $T_{L} \sim 10^{7} \mathrm{GeV}$. In this case two active neutrinos are relatively heavy, $m_{A} \sim m_{B}=($ a few $)$ $\mathrm{eV}$, so that they can constitute the hot dark matter of the Universe. Moreover, oscillations between them can solve either atmospheric or solar neutrino problem, provided that their mass splitting is small.

A variant of our scenario makes use of weakly interacting $N_{C}$ and $N_{B}$. In this case two of the usual neutrino species have masses in the range $\left(10^{-6}-10^{-1}\right) \mathrm{eV}$, and the remaining one is relatively heavy. As an example, let us take $h_{A}^{2}=5 \cdot 10^{-14}, h_{B}^{2}=10^{-15}, h_{C}^{2}<h_{B}^{2}$ and $M_{a}=20 \mathrm{GeV}$, which corresponds to the masses of usual neutrinos $m_{A} \sim 0.1 \mathrm{eV}, m_{B} \sim 2 \cdot 10^{-3} \mathrm{eV}$ and $m_{C}<m_{B}$. This variant fits particularly well into the mass pattern suggested by the solar and atmospheric neutrino data [13]. Given that $J \lesssim 10^{-2}$ due to the constraints analogous to Eq. (7), the correct value of asymmetry is obtained for $\Delta M^{2} \lesssim 10^{-2} \mathrm{GeV}^{2}$. Thus, in this case the singlet neutrinos should be degenerate in mass. The temperature of leptogenesis is lower, $T_{L} \sim 3 \cdot 10^{5} \mathrm{GeV}$. Let us note in passing that the degeneracy of masses $M_{a}$ is helpful also for obtaining the desired baryon asymmetry for very small mixing angles $\theta_{i j}$.

The crucial feature of the suggested mechanism is that it works only if Yukawa couplings of all singlet neutrinos are small, $h_{a} \sim\left(10^{-8}-10^{-6}\right)$. This smallness can be explained, e.g., by mixing of $N_{a}$ with very heavy right handed neutrinos having Yukawa couplings of the same order of magnitude as those of quarks (and charged leptons) $h_{q}$. In this case $h_{a} \sim h_{q} \sqrt{M_{a} / M_{R}}$, and for our values of $h_{a}$ and $M_{a}$ the mass scale $M_{R}$ may be close to the Grand Unification scale, $M_{R} \sim 10^{16} \mathrm{GeV}$.

We are indebted to J. Arafune and M. Fukugita for helpful discussions. This work was partly done during the ICTP Extended Workshop on Highlights in Astroparticle Physics. The work of V.R. is supported in part by the Russian Foundation for Basic Research grant 96-0217449a, and CRDF grant 649 .

[1] A. D. Dolgov, hep-ph/9707419; V. A. Rubakov and M. E. Shaposhnikov, Phys. Usp. 39, 461 (1996) (hep$\mathrm{ph} / 9603208$ ).

[2] M. Fukugita and T. Yanagida, Phys. Lett. B 174, 45 (1986).

[3] V. A. Kuzmin, V. A. Rubakov and M. E. Shaposhnikov, Phys. Lett. B 155, 36 (1985).

[4] F. R. Klinkhamer and N. S. Manton, Phys. Rev. D 30, 2212 (1984).

[5] W. Buchmüller and M. Plumacher, hep-ph/9711208; A. Pilaftsis, hep-ph/9707235; M. Flanz, E. A. Paschos, U. Sarkar and J. Weiss, Phys. Lett. B 389, 693 (1996).

[6] Our analysis can be applied with minor modifications to Dirac singlet neutrinos as well.

[7] G. Sigl and G. Raffelt, Nucl. Phys. B406, 423 (1993).

[8] Particle Data Group, Phys. Rev. D 54, 94 (1996).

[9] A. Weldon, Phys. Rev. D 28, 2007 (1983).

[10] A. Weldon, Phys. Rev. D 26, 2789 (1982).

[11] M. Luty, Phys. Rev. D 45, 455 (1992).

[12] Our results for the asymmetry are valid for any other CPsymmetric initial condition $\rho\left(t_{i}\right)$. 
[13] Y. Totsuka, Talk given at the 28th Int. Symp. on LeptonPhoton Interactions, Hamburg, Germany, 1997. 